\title{
Die Quellen der Landschaft Davos - dargestellt in Verwaltungskarten
}

\author{
Rudolf F. Zweifel
}

Am Beispiel der Landschaft Davos wird versucht, die Bedeutung der Wasserquellen für eine Siedlung aufzuzeigen. Nebst Qualität und Quantität des Wassers beeinflussen der Quellenstandort und die rechtlichen Verhältnisse den Quellwert. Dieser letzte Faktor ist Gegenstand dieses Beitrages.

Um den ganzen Einfluß einer Quelle auf die Gestaltung der Kulturlandschaft zu erfassen, ist die Kenntnis der rechtlichen Regelung unbedingte Voraussetzung. In Agrarlandschaften kann eine dynamisch, sich oft schlagartig ändernde Funktion einer erstarrten formalen Struktur gegenüberstehen. In Staaten mit einer in der Tradition wurzelnden Rechtsordnung kann andererseits ebenso hartnäckig und starr an der funktionalen wie an der formalen Struktur festgehalten werden. Im Wechselspiel dieser beiden Strukturen spielen die rechtlichen Gegebenheiten eine entscheidende Rolle. Verträge und Gewohnheitsrechte bieten daher dem Geographen eine wichtige Informationsmöglichkeit.

Das Beschaffen der Unterlagen erfolgte fast ausschließlich im Grundbuchamt. Dort sind Buchführung und Organisation genau geregelt. Trotz peinlichster Ordnung in Büchern, Karteien und Registern kann diese Form nicht völlig überzeugen. Vorab stört das Unvermögen, daß Drittpersonen innerhalb kurzer Zeit kaum einen zufriedenstellenden Uberblick über die Rechtsverhältnisse eines kleineren Gebietes erhalten können. Zwar sind die Besitzverhältnisse jedermann und sofort zugänglich, da sie schon seit eh und je kartographisch dargestellt wurden (Katasterpläne). Es fragt sich, ob nicht noch weitere im Grundbuch verankerte Informationen entsprechend graphisch dargestellt werden könnten.

\section{Grundlagen}

\subsection{Rechtliche Grundlagen}

Der Quellwert kommt in der Vielfalt der rechtlichen Regelungen um Eigentum und Nutzung der Quelle so erst recht zum Ausdruck. Bei mehrfacher Handänderung gewinnt im allgemeinen eine Quelle an Handelswert, da sie ein begehrenswertes Objekt ist. Aus diesem Grunde lohnt es sich, dem Quell- und

Wasserrecht nachzuspüren und Verträge über Kauf und Verkauf von Quellen auszuwerten. Von besonderem Reiz ist es, nicht nur die gegenwärtigen Verhältnisse zu untersuchen, sondern auch die geschichtliche Entwicklung zu verfolgen 1 . In gewissen Fällen können nur so die Zustände geklärt werden, mit denen sich der "homo faber» heute abzufinden hat. Die Verträge unserer Vorfahren sind von Gesetzes wegen zu respektieren.

Durch Volksentscheid wurde 1912 das Zivilgesetzbuch eingeführt. Der Jurist Eugen Huber (18491923) schuf damit ein Werk, das die unterschiedlichsten privaten Vertragswerke vergangener Jahrhunderte in ihrer Gesamtheit noch heute zu schützen weiß. Artikel 942 des Zivilgesetzbuches bestimmt: "Über die Rechte an den Grundstücken wird ein Grundbuch geführt.» Das Hauptbuch enthält Blätter mit fortlaufender Numerierung. Die Aufnahme des selbständigen und dauernden Rechtes in das Grundbuch geschieht durch Anlegen eines Blattes im Hauptbuch und durch die Beschreibung des Rechtes. Auf dem Blatt sind vermerkt:

Parzellennummer

Flächenausdehnung

Objekte (Ställe ...)

Name des Besitzers

Plannummer

Datum des Vertrages

Das ZGB umschreibt die einzelnen Dienstbarkeiten (Servitute). Der Begriff Wasserrecht wird juristisch nur im Zusammenhang mit Wasserrechtsverleihungen gebraucht. In dieser Arbeit dient er als Sammelbegriff für alle Servitute, die das Wasser als Gegenstand haben. Als wichtigste Servitute kommen in Frage:

Quelle (lediglich Nut-
$\quad$ zung)
Wasserbezug
Wasserfassung
Tränke
Anschluß (... an Was- serleitung oder Kanali- sation)

Jedes Servitut kann für die einzelne Parzelle ein Recht sein, wenn die Dienstbarkeit zu deren Gunsten lautet, ist aber eine Last, sobald der Eigentümer einer Quelle sich bestimmte Eingriffe eines anderen gefallen lassen muß ( $\$ 730 \mathrm{ZGB})$. Recht und Last treten in vielen Fällen gepaart auf. 


\subsection{Karten}

Als Grundlage diente der Ubersichtsplan der Landschaft Davos, Maßstab 1: 10 000. Da er jedoch nur bis 1949 nachgeführt ist, mußte vorerst der neue Zustand der Parzellierung nach den Plänen des Vermessungsbüros Davos ergänzt werden. Die hier abgedruckten Karten geben den Stand vom Frühjahr 1970 wieder. Die Parzellengrenzen sind leicht vereinfacht.

\subsection{Parzellennumerierung}

Der ungehinderte Zugang zu allen Belegen und zum Grundbuch wie auch die Einsicht in alle Vertragsdokumente vermittelten eine Reihe von vertraulichen Informationen. Aus diesem Grunde schien es ratsam, die Parzellennumerierung verschlüsselt aufzuführen. Sie erfolgt in fortlaufender Reihenfolge von 1 bis 73, entsprechend der Nummernabfolge im Grundbuch selber. Für die Belange, die hier diskutiert werden, ist die Bezifferung zweitrangig.

\section{Inventar der Servitute}

Der erste Arbeitsschritt ist die Bestandesaufnahme der Servitute, geordnet nach: a) Parzellen; b) Rechten und Lasten ${ }^{3}$.

Durch die Grundbuchbereinigung von 1952/53 für den zur Darstellung gelangten Abschnitt der Gemeinde Davos konnte fürs erste der Überblick über die Gesamtheit der Rechtsverhältnisse gewonnen werden. Die Einführung des Zivilgesetzes beabsichtigte unter anderem, alle bilateralen Verträge früherer Jahrhunderte in die Obhut des Staates zu führen und innerhalb eines vom Gesetzgeber festgelegten Rahmens die bestehenden Rechte gültig zu umschreiben. Die einzelnen Parzellenbesitzer sind über die Servitute, die ihre Liegenschaften betreffen, meist bestens orientiert, in bäuerlichen Kreisen weiß man sie sogar auswendig.

Schließlich geht es darum, daß die öffentliche Hand über die bestehenden Rechtsbeziehungen in ihrem Bann erschöpfend Kenntnis hat. Bei Streitigkeiten zwischen Kontrahenten oder Nachbarn kann sofort von Amtes wegen eingegriffen werden, um so die tatsächlichen, verbrieften Rechte sprechen zu lassen. Aber auch Planer können davon profitieren, daß im Grundbuchamt alle Informationen, die Liegenschaften betreffen, greifbar sind.

Die Inventarisation stößt auf eine Reihe von Hindernissen. Die Blattnummern im Hauptbuch stimmen nicht unbedingt mit den Parzellennummern überein. Eine spezielle Kartei hilft beim Zuordnen eines Blattes zu einer Parzelle. Aus diesem Grunde ist es vorteilhaft, zuerst den Grundkataster zu zeichnen. Somit können die Informationen aus dem Grundbuch direkt in die betreffende Parzelle eingetragen werden.

Nicht jedes Blatt im Grundbuch muß sich auf eine Parzelle beziehen. Es kann sich gleichermaßen auf mehrere Parzellen desselben Eigentümers stützen. Solche Verhältnisse bedingen bei der graphischen Darstellung eine gesonderte Behandlung.

Auf Karte 1 ist zur Orientierung nebst den Parzellennummern nur das Straßennetz angegeben. Auf weitere Angaben wird absichtlich verzichtet. Das ausgewählte Inventar der Servitute umfaßt:

Quellen

Wasserbezug

Wasserfassung

Tränke.

Ungerade Zahlen bedeuten ein Recht, gerade eine Last. Somit kann für jede Parzelle sofort abgelesen werden, wie stark sie belastet ist oder wieviele Rechte sie beanspruchen kann.

Durch einen Punktraster wird das Besitztum einer privaten Körperschaft angegeben, die mit ihren Parzellen Anteil hat, sowohl am Alpgebiet als auch am Siedlungsgebiet. Die großen Parzellen in der Nordostecke der Karte sind Alpgebiete mit einigen leistungsfähigen Quellen. Die Ziffer 2 weist darauf hin, daß diese Parzellen die eigentlichen Wasserlieferanten sind. Daraus deckt die Körperschaft einen Teil der eigenen Wasserbedürfnisse; anderseits sicherten sich die Anlieger durch Wasserbezugsrechte (Ziffer 3 ) den andern Teil der Wassermenge. Es handelt sich bei diesen Wasserbezugsrechten in der Regel um 1 "Davoserrohr», das bedeutet 10 Minutenliter Dauerlauf während des ganzen Jahres. Das Wasserrecht gilt auf ewig und wird vererbt.

Diese Menge ist für den einzelnen Bezüger beträchtlich, für den Lieferanten jedoch eine kostspielige Angelegenheit. Vorab im Winter, wenn die Quellschüttungen zum Teil erheblich zurückgehen, kann diese Wassermenge nicht immer voll garantiert werden. Meist werden in dieser Jahreszeit die Wasserhahnen Tag und Nacht ununterbrochen offen gelassen, damit keine Leitungen einfrieren. Da ganz besonders die Gemeinde Davos mit so enormen Wasserbezugsrechten belastet ist, wird dieser winterliche Engpaß zum Problem ersten Ranges. Man denke nur an die Baupolitik und an die vielen Gäste zur wasserknappen Zeit Februar und März.

Die Alpparzellen sind im besonderen dadurch gekennzeichnet, daß sie sich ein Tränkerecht gesichert haben. Diese Rechte sind in der Regel zeitlich auf die Monate Juni bis September beschränkt. Die Alpgenossenschaften mußten auf ihren eigenen Parzellen dieses Tränkerecht sichern, da für diesel- 


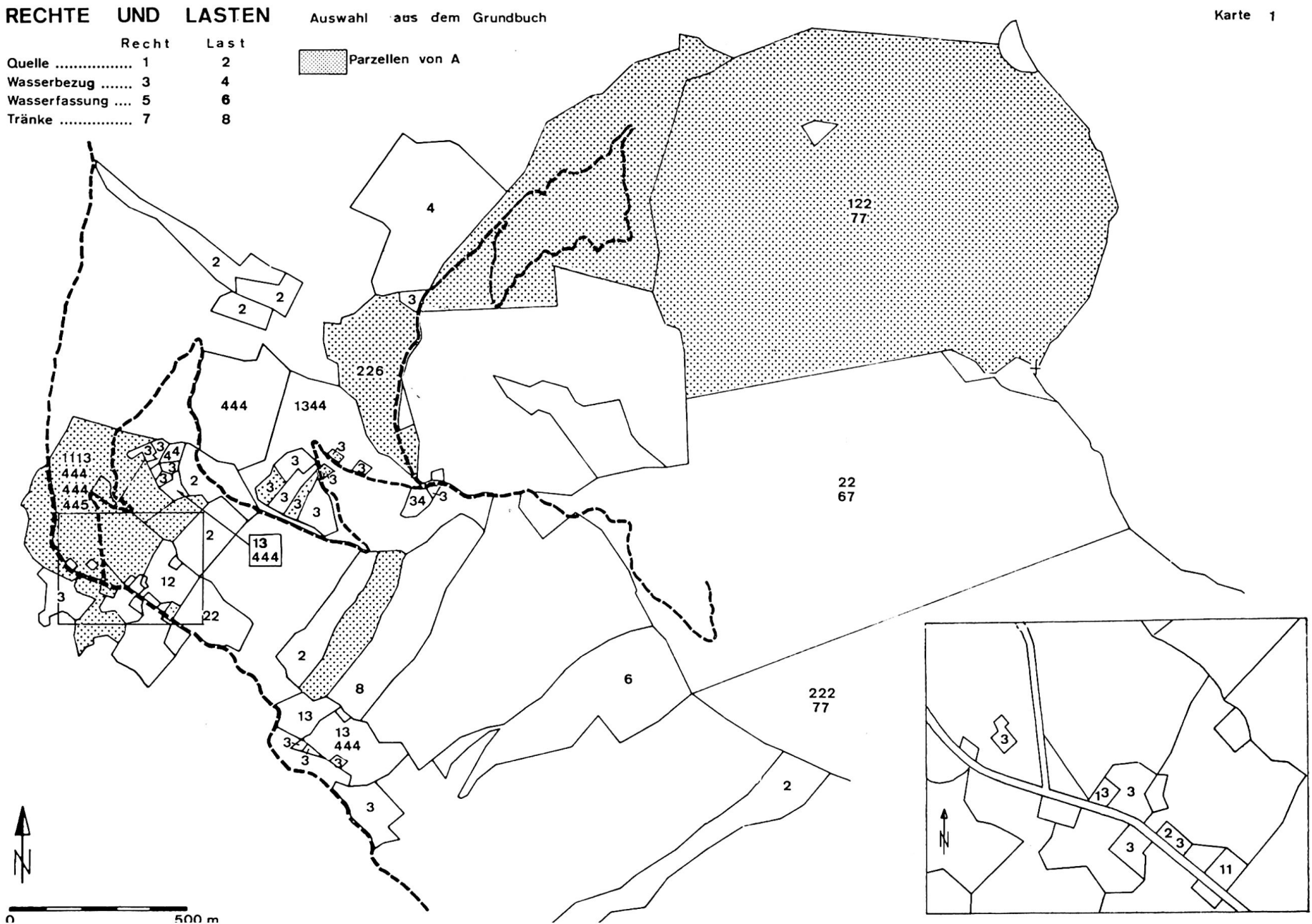


ben Gebiete das ehemals private Elektrizitätswerk eine Reihe von Wasserfassungsrechten gesichert hatte. Heute sind sie Eigentum der Gemeinde und werden nicht beansprucht.

Die Vielfalt der Servitute, die graphisch zur Darstellung gebracht werden sollten, ist hier umgangen worden. Es werden nur vier Dienstbarkeiten dargestellt, für die man mit den Zahlen 1 bis 8 auskommt. Ganz besonders müßten aber noch folgende Dienstbarkeiten berücksichtigt werden:

\section{Baurecht \\ Zufahrts- und \\ Fahrwegrecht \\ Weiderecht \\ Düngrecht \\ Holzrecht.}

Sehr oft sind die kleinen und kleinsten Parzellen, die sich mehr oder weniger mit dem Siedlungsgebiet decken, mit den meisten Servituten verbunden. Hieraus ergibt sich die Frage nach dem zu wählenden Kartenmaßstab. Der Ausweg mit einem Kärtchen, welches die kritischen Gebiete vergrößert, verschafft nur sehr beschränkt mehr Raum. Schließlich darf auch das Problem der vernünftigen Signaturwahl nicht vergessen werden, wenn alle möglichen Dienstbarkeiten zur Darstellung gelangen sollen. Eine Aufteilung in mehrere transparente Blätter scheint sich aufzudrängen. Doch darf man auch den Computer mit seiner Speicher- und neuerdings graphischen Darstellungsfähigkeit für die Lösung dieser Fragen nicht außer acht lassen.

\section{Rechtsverknüpfung der Parzellen}

Die Darstellung der Rechtsbeziehung ist der nächste Schritt. Das Schema "Wasserrecht zwischen Besitzer $A$ und seinen Kontrahenten" (Abb. 1) zeigt die Beziehungen für Quellen, Wasserbezüge und -fassungen, Durchleitungen und Tränkerechte. Auf der Ordinate sind die Parzellen des Besitzers A und auf der Abszisse die der Kontrahenten aufgetragen. Daraus können wir folgende Informationen entnehmen:

a) Gegenstand eines Vertrages können mehrere Servitute sein. Quellrecht und Tränkerecht sind oft gegenseitige Entgegenkommen der Vertragspartner. Die Bauernschaft ist bereit, das Wasser Drittpersonen abzutreten. Sie sichert sich aber für die Sommermonate ein Tränkerecht für das Vieh.

b) Besitzer A ist ein ausgesprochener Wasserlieferant. Nicht weniger als 16 Wasserbezugsrechte belasten seine Parzellen (Ed). Demgegenüber kann er die Wasserabgabe nur dadurch kompensieren, daß er die 4 Quellrechte $\left(\square^{\circ}\right), 1$ Wasserfassungsrecht $\left(F^{-}\right)$und 3 Wasserbezugsrechte $(E)$ auf fremden Parzellen für seine Bedürfnisse gesichert hatte.

c) Selbst zwischen eigenen Parzellen können Rechte verbrieft sein. Dies gilt im speziellen Fall von A für ein Quellrecht $\left(a^{a}\right)$ und zwei Wasserbezugsrechte $\left(\boldsymbol{Q}_{\bullet} \mathbf{E}\right)$.

Solche Schemas eignen sich nur für Eigentümer mit vielen Parzellen. Die Darstellung ist keine Verbesserung der bestehenden Grundbucheintragungen. Sie hat aber didaktischen Wert und erleichtert die Information. Der Grundkataster ermöglicht eine rationelle Lösung. Karte 2 zeigt das Resultat.

Der große Vorteil dieser Darstellung gegenüber den heute bestehenden Grundbucheintragungen besteht darin, daß die Angaben für den Außenstehenden leichter zugänglich und übersichtlich erfaßbar werden. Daraus könnten besonders richterliche Behörden einen Nutzen ziehen, wenn sie sich rasch und objektiv informieren wollen.

Karte 2 zeigt mit Hilfe von Linearsignaturen, mit welchen Servituten die Parzellen untereinander verbunden sind. Die Verbindungslinien geben an, zu wessen Gunsten bzw. Lasten die Dienstbarkeit lautet. Zum besseren Verständnis der Karte 2 seien in der Folge einige Beziehungen erläutert:

a) Grundbuchblätter, die sich nur auf einen Eigentümer, nicht aber auf eine Parzelle beziehen, sind schematisch eingezeichnet (Beispiele 27b, 68b, 70b). Die Plazierung dieser Rechtecke auf der Karte wurde ausschließlich durch graphische Ưberlegungen bestimmt. Aus dem gleichen Grunde ist das Blatt 27b auf zwei Rechtecke verteilt.

b) Ein seltsamer Fall tritt ein, wenn Eigentümer A Wasser einem Eigentümer $B$ abgibt und letztlich von diesem wieder erhält $(A \rightarrow B \rightarrow A)$. Dieser Fall läßt sich auf der Karte verfolgen: Parzelle 36 gehört A. Sie besitzt ein Wasserbezugsrecht zu Lasten von Parzelle 32 (gehört B). Parzelle 32 ihrerseits besitzt über das Blatt $70 \mathrm{~b}$ ein Wasserbezugsrecht zu Lasten von Parzelle 32 (gehört B). Parzelle 32 ihrerseits besitzt über das Blatt 70b ein Wasserbezugsrecht aus Parzelle 65, die dem Eigentümer A gehört. Diese Beziehung wird erst verständlich, wenn man weiß, daß B eine Genossenschaft und $A$ eines ihrer Mitglieder ist. 

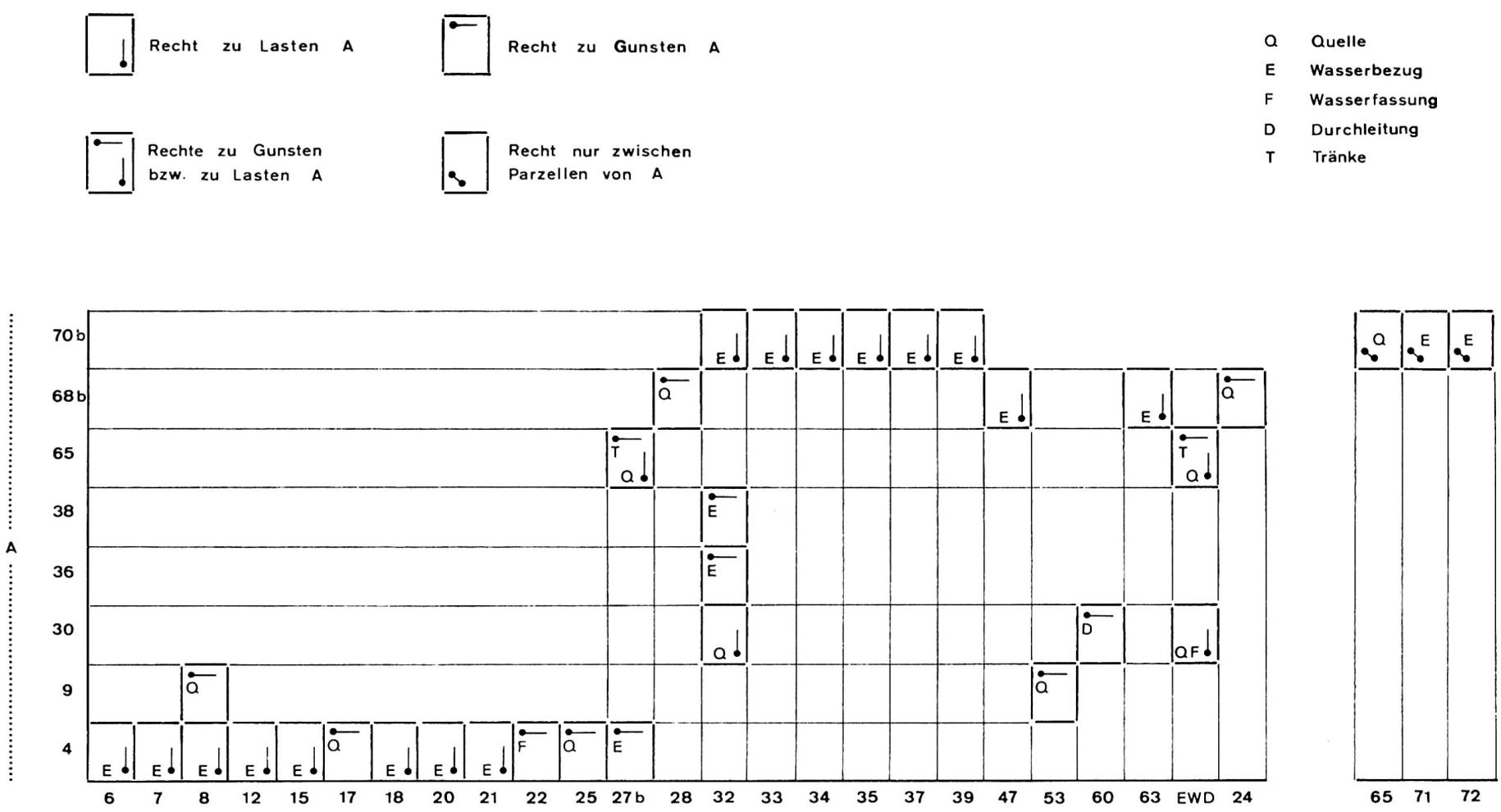

Parzellen der Kontrahenten 


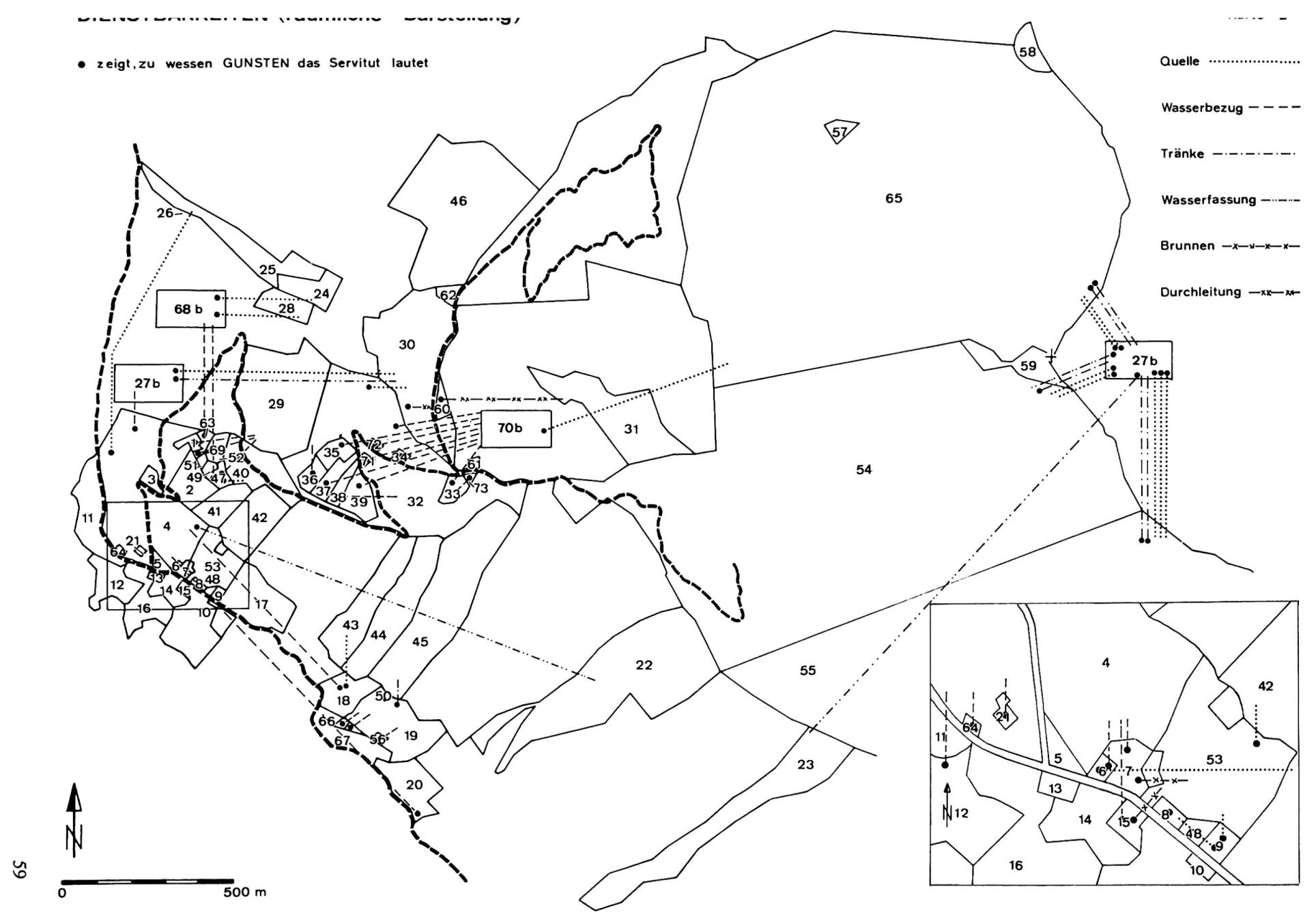


Träger der Wasserversorgung ist eine private Organisation, die bisher als Eigentümer $\mathrm{A}$ bezeichnet wurde. Dies läßt sich im Schema besonders eindrucksvoll zeigen. Aber auch auf Karte 2 kommt dies deutlich zum Ausdruck. Die Quellgebiete umfassen die Parzellen 30, 65 und 23. Letztere ist über Blatt 27b mit einer Last zu Gunsten von A versehen. Von diesen Quellgebieten wird das Wasser entweder über $70 \mathrm{~b}$ im Alpgebiet verteilt oder gelangt zur Parzelle 4. Von dort wird das Wasser gemäß Dienstbarkeitsvertrag an eine Reihe von benachbarten Parzellen verteilt (Parzellen 6, 7, 8, 12, 15, $18,20,21)$. Die Wasserlieferung erfolgt ausschließlich auf Grund der Verträge. Die ganze Wasserversorgungsanlage besitzt keine Statuten, mit denen vor allem die Unterhaltsarbeiten und deren Kostendeckung geregelt werden sollten. Diese Sachlage darf als ein Kuriosum bezeichnet werden.

Die Wassernutzung kann als eine Art Güterverteilung betrachtet werden. Damit wird das Studium der Quellen und deren Nutzung Gegenstand wirtschaftsgeographischer Untersuchungen. Zur Darstellung gelangen einerseits die Produktionsräume und anderseits die Konsum- oder Zielgebiete. Durch das Einzeichnen der ganzen Wasserversorgung, das heißt aller Leitungen und Reservoirs, kann der Transportweg als "flow chart» dargestellt werden. Diese Zeichnung wäre das Resultat der zu einem bestimmten Zeitpunkt realisierten Dienstbarkeiten. Den Planer aber interessiert vorab die Summe aller realisierbaren Beziehungen. Nicht immer sind alle theoretisch möglichen Beziehungen ausgenützt.

Karte 3 zeigt die Wasserverteilung, die nach den rechtlichen Gegebenheiten möglich ist. Im vorliegenden Falle sind alle möglichen Dienstbarkeiten praktisch genutzt. Karte 3 zeigt einen Auszug aus Karte 2. Sie zeigt die Wasserlieferanten (Eigentümer A - vergleiche Punktraster in Karte 1).

Wir unterscheiden primäre und sekundäre Wasserbezüger. Die Zahl der primären Bezüger, das heißt jener Eigentümer, die direkt mit A einen Vertrag abgeschlossen haben, hat sich in den letzten zwanzig Jahren nicht vergrößert. Doch in der Zwischenzeit haben Primärbezüger ihre Parzellen an weitere Interessenten stückweise weiterverkauft. Mit einher ging die Aufteilung der Wasserbezugsrechte, die, wie wir schon festgestellt haben, dem Einzelnen überdimensional viel Wasser zubilligen (1 Davoserrohr).

Diese Erscheinung sei an einem Beispiel erläutert: Der Eigentümer der Parzelle 47 verkaufte einen Teil seines Bodens. Es entstanden dadurch die Par- zellen 49, 51 und 52. Alle drei Neubesitzer erhielten Anteil am einzigen Davoserrohr der Parzelle 47. Diese sekundären Wasserbezüger gaben sich stillschweigend mit einer kleineren Wassermenge zufrieden. Für A ist dies wichtig, da er letztlich alleine die Verantwortung trägt, d. h. auch in den strengsten Wintertagen die knappen Anteile am Davoserrohr zu garantieren hat. Das Verteilerschema sieht vereinfacht so aus:

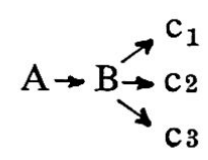

Karte 3 zeigt nur eine Möglichkeit, wie Karte 2 zu interpretieren ist. Ähnliche Darstellungen könnten für eigene und fremde Quellen gefunden werden; Durchleitungsrechte; Tränkerechte.

\section{Zusammenfassung}

Es erstaunt, daß Rechtskarten dieser Art kaum existieren. Doch erst mit der graphischen Darstellung wird es möglich, Rechtszusammenhänge einzelner Parzellen untereinander oder zueinander rasch zu erkennen. An solchen Karten muß die Geographie interessiert sein. Die Kulturlandschaft wird auch durch die rechtlichen Verhältnisse mitgeprägt.

Die Geschichte lehrt uns, alles Anthropogene in stetem Wandel zu sehen. Beispielsweise siedelten die Walser anfänglich weit zerstreut in der Landschaft Davos. Dies bedeutet aber nicht, daß sie keine gemeinsamen Interessen an Quellen oder Wasserläufen geltend machten. Schon im 14. und 15. Jahrhundert fanden Wasserrechtshändel statt, bei denen die Obrigkeit der Landschaft Davos einzugreifen hatte 4 . Im Laufe der Jahrhunderte vermehrten sich die Rechtsbeziehungen, weil immer mehr Menschen sich ansiedelten. Die letzten Jahrzehnte beschleunigten diesen Prozeß. Die Interessen am Wasser nahmen sprunghaft zu.

Außer den Bedürfnissen für Siedlungen und Landwirtschaft treten in immer stärkerem Maße die Forderungen von Industrie und Tourismus hinzu. Bald wird das gesunde Quellwasser aus den Alpgebieten zum Exportgut werden, wenn in den dicht besiedelten Gebieten der Mangel an gutem Trinkwasser zunimmt ${ }^{5}$. Dann dürfte auch für die Alpgebiete der Moment gekommen sein, wo ihre sämtlichen Wasserreserven genutzt werden müssen. Und genau aus diesem Grunde ist es wichtig, wenn die Rechtsgrundlagen genauestens bekannt und rasch zugänglich sind. 


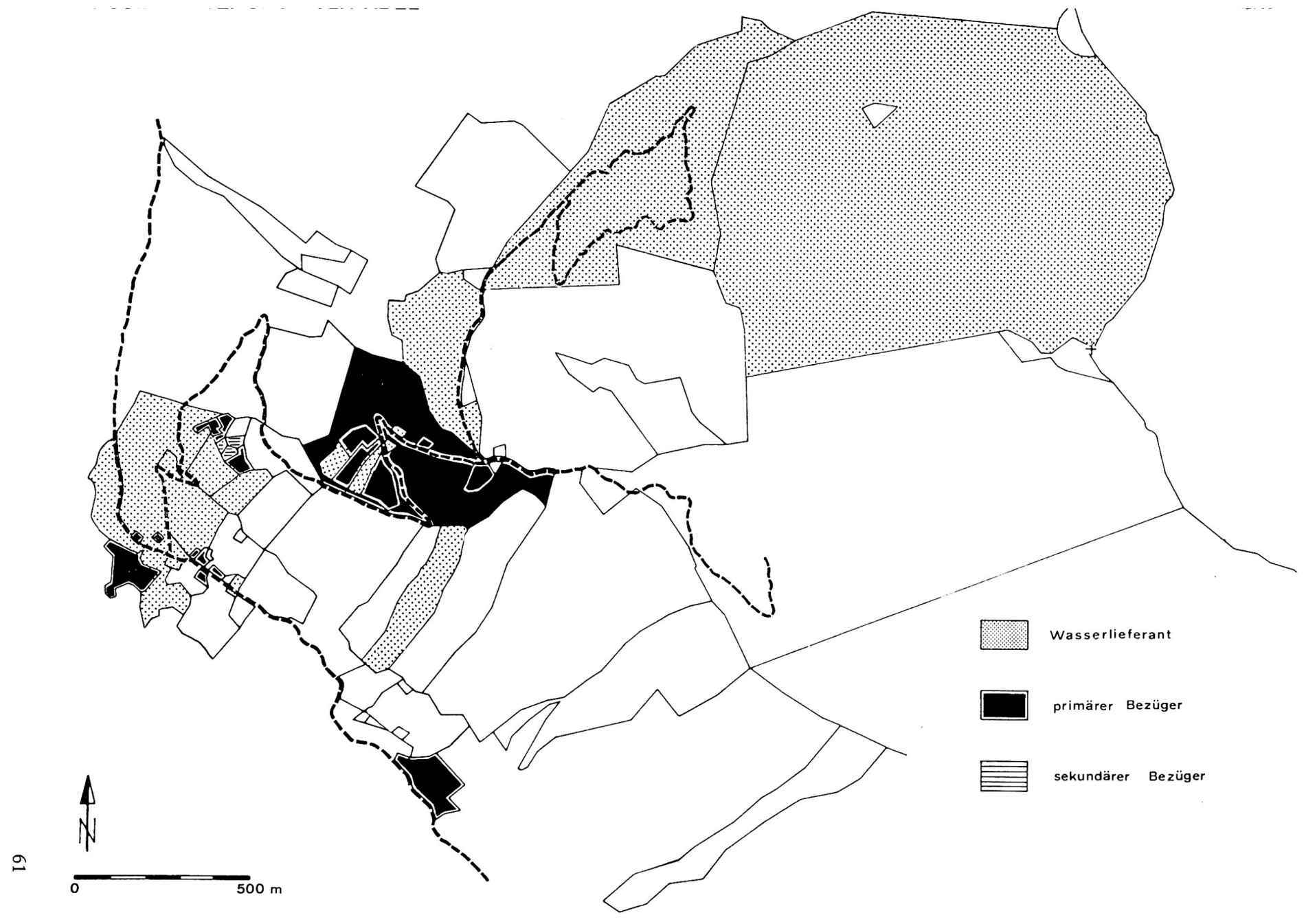




\section{Anmerkungen und Literatur}

1 Aus Platzgründen muß auf eine ausführliche Darstellung historischer Tatsachen verzichtet werden. Diese sind in der Diplomarbeit (R. F. Zweifel, Geographisches Institut der Universität Zürich 1970) zusammengetragen.

2 Das Quellrecht läßt das Eigentum an Quellen unberührt. Es ist rechtlich nicht vorgesehen, den Begriff Quellkauf zu postulieren. Die Quelle gehört gemäß § 667 bzw. 704 ZGB zum Eigentum an Grund und Boden.
3 Das Inventar ist in der vertraulichen Dokumentation B zur Diplomarbeit tabellarisch zusammengetragen.

${ }^{4}$ Nach Urkunden des Gemeindearchivs in Davos Platz. Diese sind zum Teil in der Diplomarbeit wiedergegeben.

5 Frei zitiert nach Prof. Dr. Jaag, Abschiedsvortrag an der ETH, am ETH-Tag 1970.

Für die ungehinderte Einsichtnahme in die Grundbuchunterlagen sei der Gemeindebehörde von Davos herzlich gedankt.

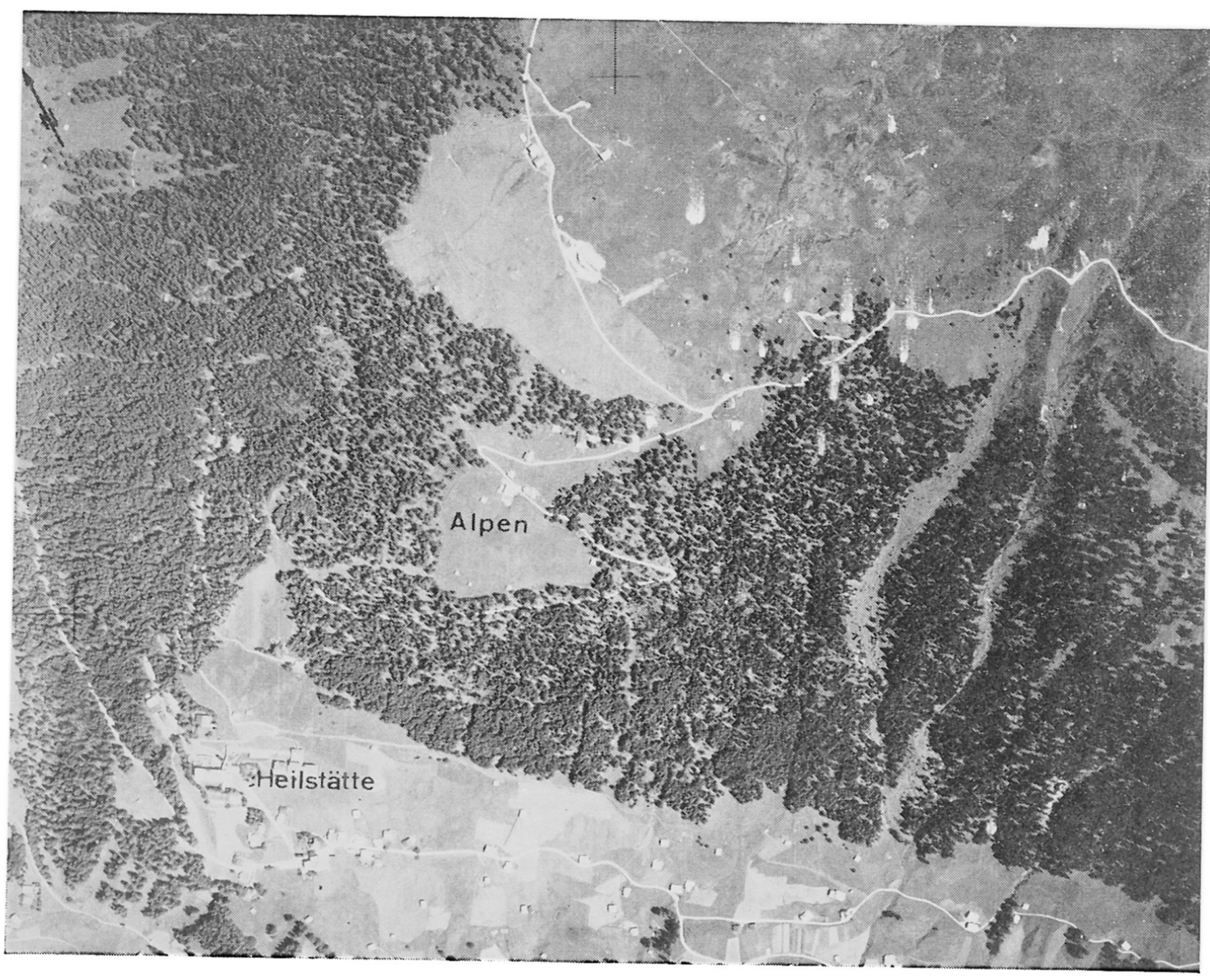

Luftbild Davos Clavadel mit Heilstätte und Clavadeler Alpen. Karten und Luftbild können mit Hilfe des Wegnetzes verglichen werden. Aufnahme durch Luftaufklärungsdienst Dübendorf am 15. 7. 1969, 11.15 Uhr. (Das Luftbild wurde vom Geographischen Institut der Universität Zürich, Prof. Dr. H. Häfner, zur Verfügung gestellt.) Maßstab des Luftbildes ca. 1: 15000 